\title{
EL AUTOCONCEPTO EN LA ETAPA DE EDUCACIÓN PRIMARIA: INCIDENCIA EN LOS ALUMNOS MÁS CAPACES
}

\author{
Ramón García Perales \\ Universidad de Castilla-La Mancha (España)

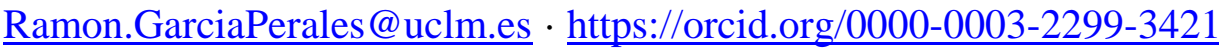 \\ Inmaculada Canuto González \\ Universidad de Castilla-La Mancha (España) \\ Inmaculada.Canuto@alu.uclm.es \\ Ascensión Palomares Ruiz \\ Universidad de Castilla-La Mancha (España) \\ Ascension.Palomares@uclm.es · https://orcid.org/0000-0003-3350-2341
}

\begin{abstract}
Resumen. Los nuevos planteamientos en el campo de las altas capacidades, frente al tópico de considerar únicamente la inteligencia como elemento fundamental en su identificación, optan por un amplio abanico de cualidades y dimensiones a tener en cuenta en la detección. Entre ellas, aparece el autoconcepto entendido como la autoimagen mental del sujeto. Este constructo y su relación con otras variables, caso del rendimiento académico o el sexo de los escolares, podrían ser factores influyentes en las cifras llamativamente bajas de diagnóstico de alumnado con altas capacidades y en los resultados negativos en evaluaciones internacionales de rendimiento. Para comprobar la relación entre estas variables, hemos realizado una investigación cuantitativa para evaluar el autoconcepto general y académico en una muestra de 118 sujetos de $4^{\circ}, 5^{\circ}$ y $6^{\circ}$ de Educación Primaria, incluidos escolares con altas capacidades diagnosticadas. Se ha utilizado la Escala de Autoconcepto de Piers Harris. Los resultados arrojan una correlación positiva entre el autoconcepto general y académico y el rendimiento académico, no encontrándose relación entre autoconcepto y género, alta capacidad, edad y curso. De esta forma, se deberán favorecer, desde los propios procesos de enseñanza y aprendizaje, actuaciones específicas en pro de la mejora del autoconcepto del alumnado teniendo presente su rendimiento académico. El bienestar de los escolares deberá de ser una premisa fundamental de la praxis docente.
\end{abstract}

Palabras clave: Autoconcepto, altas capacidades, género, rendimiento académico, escuela.

\section{THE SELF-CONCEPT IN THE STAGE OF PRIMARY EDUCATION: INCIDENCE IN THE MOST CAPABLE STUDENTS}

Abstract. The new approaches in the field of high capacities, compared to the topic of considering only intelligence as a fundamental element in their identification, opt for a wide range of qualities and 
dimensions to be taken into account in detection. Among them, self-concept appears as the mental selfimage of the subject. This construct and its relationship with other variables, such as the academic performance or the sex of schoolchildren, could be influential factors in the strikingly low figures for the diagnosis of students with high abilities and negative results in international performance evaluations. To verify the relationship between these variables, we conducted a quantitative research to evaluate the general and academic self-concept in a sample of 118 subjects of 4th, 5th and 6th grade of Primary Education, including school children with high diagnostic abilities. The Piers Harris Self-concept Scale has been used. The results show a positive correlation between general and academic self-concept and academic performance, not finding a relationship between self-concept and gender, high ability, age and course. In this way, specific actions should be favored, from the teaching and learning processes themselves, in favor of improving the students' self-concept, keeping in mind their academic performance. The welfare of schoolchildren should be a fundamental premise of the teaching practice.

Keywords: Selfconcept, high capacities, gender, academic performance, school.

\section{Introducción}

Las puntuaciones obtenidas por el alumnado español en distintas evaluaciones internacionales que tratan de medir su rendimiento educativo son bajas en comparación con el promedio de los países de la Organización para la Cooperación y el Desarrollo Económico (OCDE). Tomamos como referencia los informes PISA (Program for International Student Assessment) desarrollados por la OCDE, dada la importancia y repercusión de sus conclusiones en las políticas educativas y la elevada tasa de participación de distintos países (García, 2014). En las seis ediciones desarrolladas hasta la fecha (2000, 2003, 2006, 2009, 2012 y 2015), han evaluado la competencia lectora, matemática y científica. En todas las ediciones y para todas las competencias, España ha puntuado por debajo de la media de la OCDE, aunque la brecha ha ido estrechándose a lo largo de los sucesivos informes (OCDE, 2005a, 2005b, 2007, 2010, 2014, 2016). Además resulta reseñable el hecho de que en España apenas existen alumnos strong performers y top performers, es decir, alumnos de rendimiento excelente en PISA (García, Silva, y Viseu, 2017).

Por otro lado, en España también son significativamente bajas las cifras de diagnóstico de altas capacidades. Según Jiménez y García (2013), deberían considerarse alumnos más capaces al menos entre el 5 y $10 \%$ de la población escolar. Sin embargo, los últimos datos publicados por el Ministerio de Educación, Cultura y Deporte para el curso 2016/2017, último del que se disponen de datos para enseñanzas no universitarias, señalan que el número de alumnos españoles diagnosticados con altas capacidades intelectuales supone solamente el $0,33 \%$ del total del alumnado, tal y como se observa en la Tabla 1. 
Tabla 1

Prevalencia de casos de alta capacidad en España en el curso 2016/2017

\begin{tabular}{|c|c|c|}
\hline CC. AA. & N. ${ }^{o}$ total de alumnos con & \\
\hline Curso 2016/2017 & alta capacidad & $\%$ \\
\hline Andalucía & 11.582 & 0,72 \\
\hline Aragón & 182 & 0,08 \\
\hline Principado de Asturias & 804 & 0,59 \\
\hline Illes Balears & 831 & 0,46 \\
\hline Canarias & 2.122 & 0,61 \\
\hline Cantabria & 128 & 0,14 \\
\hline Castilla y León & 638 & 0,18 \\
\hline Castilla-La Mancha & 411 & 0,11 \\
\hline Cataluña & 417 & 0,03 \\
\hline Comunitat Valenciana & 1.063 & 0,12 \\
\hline Extremadura & 266 & 0,15 \\
\hline Galicia & 1.590 & 0,40 \\
\hline Comunidad de Madrid & 2.190 & 0,19 \\
\hline Región de Murcia & 3.698 & 1,27 \\
\hline Comunidad Foral de Navarra & 399 & 0,36 \\
\hline País Vasco & 536 & 0,14 \\
\hline Rioja & 274 & 0,50 \\
\hline Ceuta & 2 & 0,01 \\
\hline Melilla & 0 & 0,00 \\
\hline España & 27.133 & 0,33 \\
\hline
\end{tabular}

Nota: Elaboración propia a partir de datos del Ministerio de Educación Cultura y Deporte -MECD- (2018)

En este punto, cabe hacer mención a las nuevas corrientes en el campo de las altas capacidades que, ante el tópico de considerar únicamente la inteligencia como elemento fundamental en su identificación, optan por tener en cuenta un amplio abanico de cualidades y dimensiones (Pfeiffer, 2015; Stoeger, Balestrini, y Ziegler, 2018). De este modo, adquieren relevancia en la situación planteada variables como las expectativas, las aspiraciones personales o el autoconcepto, entre otras, debiendo tener en cuenta además las diferencias habidas entre sexos.

El término autoconcepto es complejo y difícil de reconocer y de aislar de otras percepciones mentales del ser humano, caso por ejemplo de la autoestima. Pero es importante distinguir el uno del otro ya que, aunque a veces son utilizados indistintamente, existen diferencias que han de ser tenidas en cuenta. El autoconcepto se corresponde con una dimensión cognitiva de nuestra propia imagen mental, puesto que es una información que creamos sobre nosotros mismos pero que deja de lado cualquier juicio de valor y carece, por tanto, de componente moral (Zeidner, y Shani-Zinovich, 
2015). Sin embargo, aunque vinculada al autoconcepto, la autoestima está cargada de un fuerte componente emocional y una valoración subjetiva. Es decir, el autoconcepto es una serie esquemas mentales sobre nosotros mismos al que le adjudicamos una valoración positiva (autoestima positiva) o negativa (autoestima negativa). Además, a partir de la profundización conceptual desarrollada por distintos investigadores (caso de Harter y Byrne, citados en Ancillo et al., 2001), el autoconcepto se puede fragmentar en distintos componentes, como son el autoconcepto conductual, académico, social, físico, etc.

Como decíamos, el autoconcepto, junto a las expectativas personales y sin perder de vista el género, podría ayudar a explicar el bajo rendimiento académico y las tasas de diagnóstico de altas capacidades en España. En este sentido, son muchos los autores que defienden que la adolescencia es una etapa crucial (Jiménez, 2014; Kerr, 1999; Trillo, 2012). En la infancia las niñas altamente capaces muestran expectativas similares a sus pares varones, buscan el éxito académico y son competitivas en la misma medida que ellos. Sin embargo, a partir de esta etapa se dan una serie cambios motivados por la aculturación, principalmente, que hacen descender su nivel de aspiraciones y de consecución del éxito durante la adolescencia y la adultez (Kerr, 1999). Es en la juventud cuando empieza a disminuir su confianza en sí mismas y en sus capacidades, aumentándose los niveles de inseguridad. Y es que las chicas más capaces tienen una percepción diferente de su capacidad y de su éxito académico con respecto a los chicos (Al-Srour y Al-Oweidi, 2016), tendiendo a relacionarlos con la suerte (Jiménez, 2014).

Este autoconcepto de las alumnas más capaces afecta irremediablemente a su rendimiento académico. Así lo certifica el informe de la OCDE ¿Qué subyace bajo la desigualdad de género en educación?, que indica que "de media, en los países de la OCDE, la diferencia en puntuación en matemáticas entre los chicos y chicas de mejor rendimiento es de 19 puntos. Sin embargo, si comparamos chicas y chicos que dijeron tener un nivel similar de confianza en sí mismos con respecto a las matemáticas, la brecha de género desaparece" (OCDE, 2015, p. 2).

$\mathrm{Y}$ es que según este informe las chicas, en general, tienen menos autoconfianza para resolver problemas matemáticos. Pero cuando muestran tener confianza, los resultados se igualan a los hombres, además de mostrar mayores expectativas. Así, continúan afirmando que "esta diferencia de género podría relacionarse con la confianza que los alumnos tengan en sí mismos. Al tener más confianza, se dan más libertad para equivocarse, para practicar procesos de prueba y error que resultan fundamentales para adquirir mayor conocimiento y comprensión de las matemáticas o de las ciencias" (OCDE, 2015, p. 3).

En esta misma dirección apuntan los resultados y conclusiones de distintas investigaciones que concluyen que el autoconcepto es una de las variables de mayor incidencia en el rendimiento académico (Naranjo, 2007; Navarro, Tomás, y Oliver, 2006; Rodríguez, 1982). Además, se ha demostrado que resulta necesario considerar el género como una variable importante en los estudios que investigan este constructo (Ancillo et al., 2001), pues las diferencias en este sentido están avaladas por distintas investigaciones (Amezcua, y Pichardo, 2000; Padilla, García, y Suárez, 2010; Pastor, Balaguer, y García-Merita, 2003).

En definitiva, según la literatura aportada, tanto el desempeño académico como las tasas diagnóstico de altas capacidades y las desigualdades de género existentes, se deberían en gran medida al autoconcepto y las perspectivas y percepción de las propias 
capacidades. Es por ello que con esta investigación se pretende clarificar la incidencia del autoconcepto en base a distintas variables de investigación.

\section{Método}

Nuestra investigación parte de consideraciones, ya señaladas en el apartado anterior, como los resultados por debajo del promedio de la OCDE en evaluaciones internacionales de rendimiento, incluyendo el número limitado de alumnos strong y top performers, o las tasas mínimas de diagnóstico de altas capacidades intelectuales entre la población escolar. Debido a ello, nos surge el interrogante de hasta qué punto tiene incidencia el autoconcepto del alumnado. De esta manera, según la bibliografía consultada, partimos de la hipótesis de que el autoconcepto es una variable que correlaciona positivamente con el rendimiento académico y, además, niveles bajos de este constructo pueden incidir en la detección e intervención educativa con alumnado de altas capacidades.

Para probar o desechar la hipótesis, se ha llevado a cabo una investigación cuantitativa con un análisis de resultados descriptivos y diferenciales, en función de distintas variables de investigación, y se han extraído las conclusiones más importantes en cuanto a las implicaciones que podrían derivarse para las políticas educativas y la práctica docente, siempre buscando la mejora del autoconcepto del alumno.

Para ello, el objetivo establecido para esta investigación ha sido valorar el autoconcepto general y académico en una muestra de alumnos y alumnas de los niveles de $4^{\circ}, 5^{\circ}$ y $6^{\circ}$ de Educación Primaria, comparando posteriormente los resultados de acuerdo a distintas variables, caso por ejemplo de la existencia de altas capacidades intelectuales diagnosticadas.

\section{Muestra}

En la investigación han participado 118 alumnos de diferentes centros escolares de Educación Infantil y Primaria seleccionados por medio de un muestreo probabilístico de tipo aleatorio. Los niveles utilizados de la etapa de Educación Primaria han sido $4^{\circ}$ con 40 alumnos y alumnas, $5^{\circ}$ con 30 escolares y $6^{\circ}$ con 48 estudiantes. Entre este alumnado, aparecen 21 identificados con altas capacidades intelectuales por parte de los Servicios de Orientación Educativa que atienden a sus centros escolares.

\section{Variables}

La variable principal de la investigación ha sido el autoconcepto, de tipo general y académico. El primero de ellos es entendido como el conjunto de atribuciones, ideas y creencias que cada persona tiene sobre sí misma. El segundo, es una subdimensión del anterior y se define como la percepción del alumno acerca de su propia capacidad para llevar a cabo determinadas tareas académicas e intelectuales.

Por otro lado, se han utilizado otras variables como:

- Altas capacidades intelectuales detectadas, tal y como se ha señalado anteriormente, contamos con 21 escolares identificados como tal. Junto al autoconcepto, se conforma como variable fundamental de la investigación.

- Sexo, distinguiendo entre Hombre o Mujer. En este estudio, 67 han sido de sexo masculino y 51 de sexo femenino. 
- Rendimiento académico, definido a fecha de junio de 2018 a través de la calificación media en todas las áreas curriculares y cuantificándose en rendimiento Alto (calificaciones medias de Sobresaliente, 40 alumnos y alumnas), Medio (calificaciones medias de Notable y Bien, 50 escolares) y Bajo (calificaciones medias de Suficiente e Insuficiente, 28 alumnos y alumnas).

- $\quad$ Edad, utilizando la edad cronológica del alumnado en el momento de la investigación, que va desde los 9 a los 12 años $(25,36,34$ y 23 escolares respectivamente).

- $\quad$ Curso, repartida la muestra entre $4^{\circ}, 5^{\circ}$ y $6^{\circ}$ nivel de Educación Primaria (40, 30 y 48 alumnos y alumnas respectivamente).

\section{Instrumento}

El instrumento principal administrado para la evaluación del autoconcepto ha sido la Escala de Autoconcepto de Piers Harris. La versión empleada es la traducción al castellano usada en el estudio La evaluación de la adaptación socioemocional a través del autoinforme. El autoconcepto y la empatía (Díaz-Aguado, y Martínez-Arias, 1995).

Esta escala está diseñada para niños de entre 7 y 12 años aproximadamente, y se compone de 80 afirmaciones con respuesta dicotómica de "Sí o No". De estos ítems, 36 son positivos y 44 negativos. Las afirmaciones positivas se puntúan con 1 la respuesta "Sí" y con 0 la respuesta "No"; en los ítems negativos se procederá al contrario. La suma de las puntuaciones obtenidas permite calcular tanto el autoconcepto general (de 0 a 80 puntos), como de cada una de las seis áreas siguientes: estatus intelectual, autoconcepto académico (de 0 a 17 puntos, utilizado en esta investigación), comportamiento, ansiedad, popularidad, aspecto corporal y cualidades físicas, felicidad y satisfacción.

Según el estudio de Díaz-Aguado y Martínez-Arias (1995), el índice de fiabilidad Alpha de Cronbach del autoconcepto general es de ,90 y el del autoconcepto académico de ,79, llegando este último a ,90 en otras investigaciones (caso de Guillén, y Ramírez, 2011). Además, son más los autores que avalan la fiabilidad de la escala a nivel global, con coeficientes Alpha de Cronbach que oscilan entre ,83 y ,98 (Epstein, 1985; Puigcerver, 2003).

\section{Procedimiento}

El primer paso fue la puesta en contacto con los directores y directoras de varios centros educativos para solicitar su participación en esta investigación. Posteriormente, se demandó la autorización por escrito a las familias de los grupos de escolares cuyos centros y docentes mostraron una disposición favorable a formar parte de este estudio. En la selección de centros se buscaron escuelas que tuvieran escolarizados entre su alumnado a estudiantes con altas capacidades intelectuales.

Tras seleccionar la muestra, se administró el instrumento señalado anteriormente de forma grupal. De forma paralela, se demandó a los tutores que indicasen qué rendimiento académico medio en el conjunto de las áreas curriculares tenían cada alumno y cada alumna con fecha de junio de 2018. Por último, para el manejo de los datos obtenidos se ha hecho uso del programa estadístico IBM SPSS Statistics en su versión 22.0, las pruebas utilizadas y las relaciones establecidas entre las variables se muestran en el apartado siguiente. 


\section{Resultados}

El desarrollo de este apartado está conformado por los siguientes subapartados:

- Resultados alcanzados para la variable autoconcepto: general y académico.

- $\quad$ Resultados en función de altas capacidades detectadas.

- Resultados en función del resto de variables incluidas en la investigación.

Resultados alcanzados para la variable autoconcepto: general y académico

Para cada tipo de autoconcepto y con vistas al desarrollo de los análisis posteriores, se han aglutinado las puntuaciones en tres niveles (nivel 1 bajo, nivel 2 medio y nivel 3 alto) tanto para el autoconcepto general como académico. Sus frecuencias y porcentajes aparecen señalados en la Tabla 2.

Tabla 2

Frecuencias y porcentajes para cada nivel para las variables autoconcepto general y autoconcepto académico

\begin{tabular}{lcccccc}
\hline \multirow{2}{*}{ Niveles } & \multicolumn{3}{c}{ Autoconcepto general } & \multicolumn{3}{c}{ Autoconcepto académico } \\
\cline { 2 - 7 } & Intervalo & f & $\%$ & Intervalo & f & $\%$ \\
\hline Bajo & $30-45$ & 13 & 11,0 & $4-8$ & 17 & 14,4 \\
Medio & $46-61$ & 38 & 32,2 & $9-12$ & 41 & 34,7 \\
Alto & $62-77$ & 67 & 56,8 & $13-17$ & 60 & 50,9 \\
\hline
\end{tabular}

De acuerdo a los resultados reflejados en estas dos tablas, los niveles de autoconcepto general y académico de los participantes se han ubicado mayoritariamente en el nivel alto, $56,8 \%$ y 50,8\% respectivamente, frente al $11,0 \%$ de uno y $14,4 \%$ para el otro dentro del nivel bajo. La media de los resultados para los tres niveles ha sido de 2,46 para el autoconcepto general, desviación típica de ,69, y de 2,36 para el autoconcepto académico, desviación típica de ,72.

\section{Resultados en función de altas capacidades detectadas}

El estudio de los resultados para cada uno de los tipos de autoconcepto en función de la existencia o no de altas capacidades detectadas, constituye la base del desarrollo de esta investigación. Su importancia ha quedado manifiesta en la fundamentación teórica del presente artículo.

En primer lugar, se han analizado los resultados para el autoconcepto general en función de la alta capacidad detectada, mostrándose en la Tabla 3 las frecuencias y porcentajes. En ella, los alumnos con altas capacidades muestran una frecuencia elevada en el nivel 1 y 2 , mientras que aquellos alumnos no detectados como tal aparecen mayoritariamente en el nivel 3 o alto. La media por los alumnos que sí están diagnosticados ha sido de 2,05, desviación típica de ,74, para los que no de 2,55, desviación típica de ,65. A pesar de la discrepancia de la muestra entre ambos grupos para esta variable, sí 21 estudiantes y no 97 estudiantes, las diferencias en los promedios obtenidos hacen imprescindible continuar con el análisis estadístico. Así, en la prueba Chi-cuadrado, valor de 9,26 significatividad de ,01, se han observado diferencias estadísticamente significativas en la relación entre las variables alta capacidad detectada 
y autoconcepto general. El índice de correlación de Pearson entre ambas variables ha sido de -,28, con una significatividad de ,00. Queda demostrado que existe significatividad entre variables, es decir, hay relación entre bajo autoconcepto general y existencia de altas capacidades intelectuales. Por otro lado, tras relacionar autoconcepto general, alta capacidad detectada y sexo, aparece un índice de correlación de Pearson de ,- 13 , no existiendo significatividad al ser el valor de la misma de ,17.

Tabla 3

Frecuencias y porcentajes para la variable alta capacidad detectada según cada nivel definido para la variable autoconcepto general

\begin{tabular}{lcccc}
\hline \multicolumn{1}{c}{ Niveles } & \multicolumn{2}{c}{ Sí } & \multicolumn{3}{c}{ No } \\
\hline \multirow{3}{*}{ Bajo } & $\mathbf{f}$ & $\boldsymbol{\%}$ & $\mathbf{f}$ & $\boldsymbol{\%}$ \\
Medio & 5 & 4,24 & 8 & 6,78 \\
Alto & 10 & 8,47 & 28 & 23,73 \\
\hline
\end{tabular}

Por otra parte, se pasa a analizar el autoconcepto académico con la variable alta capacidad detectada, señalando en la Tabla 4 las frecuencias y porcentajes. Estos alumnos con altas capacidades muestran, atendiendo a la proporción de la muestra de uno y otro grupo, resultados similares para cada uno de los niveles definidos. En ambos grupos, el mayor número de alumnos se ubica en el 3 o alto. La media por los alumnos que sí están diagnosticados ha sido de 2,38, desviación típica de ,67, para los que no de 2,36, desviación típica de ,74. Como puede observarse, los promedios de ambos grupos son muy similares. Esta tendencia se observa en la pruebas Chi-cuadrado, valor de ,96 significatividad de ,62, y en el cálculo del índice de correlación de Pearson, valor de ,01 y significatividad de, 91 , en las que se obtienen índices no significativos entre variables. Tampoco aparece significatividad si incluimos la variable sexo en esta relación. Por tanto, se puede concluir que no existe significatividad entre autoconcepto académico y alta capacidad detectada.

Tabla 4

Frecuencias y porcentajes para la variable alta capacidad detectada según cada nivel definido para la variable autoconcepto académico

\begin{tabular}{lcccc}
\hline \multicolumn{1}{c}{ Niveles } & \multicolumn{2}{c}{ Sí } & \multicolumn{3}{c}{ No } \\
\hline \multirow{3}{*}{ Bajo } & $\mathbf{f}$ & $\boldsymbol{\%}$ & $\mathbf{f}$ & $\%$ \\
Medio & 2 & 1,70 & 15 & 12,71 \\
Alto & 9 & 7,63 & 32 & 27,12 \\
\hline
\end{tabular}

\section{Resultados en función del resto de variables incluidas en la investigación}

En este apartado, se muestran los resultados para las siguientes variables: sexo, rendimiento académico, edad y curso escolar. 
En cuanto al sexo, en la Tabla 5 se muestran los resultados para cada nivel de autoconcepto general, siendo el nivel 3 o alto en el que se encuentra la mayoría de escolares para ambos sexos. En el nivel 1 o bajo aparecen 8 escolares de sexo masculino, 6,78\% de la muestra total, y 5 escolares de sexo femenino, 4,24\% de la muestra total. La media de los escolares de sexo masculino ha sido de 2,52, desviación típica de ,70, mientras que para los estudiantes de sexo femenino la media ha sido de 2,37, desviación típica de ,66. Por otro lado, de acuerdo a la prueba Chi-cuadrado, valor de 35,82 significatividad de ,43, e índice de correlación de Pearson, valor de -,10 significatividad de ,31, no aparecen diferencias estadísticamente significativas. Además, tal y como se ha señalado con anterioridad, no existe relación entre autoconcepto general, sexo y alta capacidad detectada.

Tabla 5

Frecuencias y porcentajes para la variable sexo según cada nivel definido para la variable autoconcepto general

\begin{tabular}{lcccc}
\hline \multicolumn{1}{c}{ Niveles } & \multicolumn{2}{c}{ Hombre } & \multicolumn{2}{c}{ Mujer } \\
\hline \multirow{3}{*}{ Bajo } & $\mathbf{f}$ & $\boldsymbol{\%}$ & $\mathbf{f}$ & $\boldsymbol{\%}$ \\
\cline { 2 - 5 } Medio & 8 & 6,78 & 5 & 4,24 \\
Alto & 16 & 13,56 & 22 & 18,64 \\
\hline
\end{tabular}

En la relación entre autoconcepto académico y sexo, Tabla 6, se observa equilibrio en cuanto a los resultados alcanzados para ambos sexos, destacando la igualdad en el nivel 2, a pesar de la diferencia de muestra, con 20 alumnos, 16,95\%, y 21 alumnas, 17,79\%. La media de los escolares de sexo masculino ha sido de 2,34, desviación típica de 77 , mientras que para los estudiantes de sexo femenino la media ha sido de 2,39, desviación típica de ,67. Por otro lado, de acuerdo a la prueba Chicuadrado, valor de 14,71 significatividad de ,40, e índice de correlación de Pearson, valor de -,06 significatividad de ,55, no aparecen diferencias estadísticamente significativas.

Tabla 6

Frecuencias y porcentajes para la variable sexo según cada nivel definido para la variable autoconcepto académico

\begin{tabular}{lcccc}
\hline \multicolumn{1}{c}{ Niveles } & \multicolumn{2}{c}{ Hombre } & \multicolumn{3}{c}{ Mujer } \\
\hline \multirow{3}{*}{ Bajo } & f & \% & f & \% \\
Medio & 12 & 10,17 & 5 & 4,24 \\
Alto & 20 & 16.95 & 21 & 17,79 \\
\hline
\end{tabular}

La siguiente variable de análisis ha sido el rendimiento académico. En la Tabla 7 se muestran los resultados en función de los niveles fijados para el autoconcepto general, observándose un equilibrio generalizado en los porcentajes a excepción del 
nivel bajo de rendimiento y nivel alto de autoconcepto general. La media de los escolares de rendimiento bajo ha sido de 2,18, desviación típica de ,72, rendimiento medio 2,56, desviación típica de ,64, y rendimiento alto 2,53, desviación típica ,68. La prueba Chi-Cuadrado, valor de 93,43 significatividad de ,03, e índice de correlación de Pearson, valor de -,33 significatividad de ,00, reflejan diferencias estadísticamente significativas en la relación entre autoconcepto general y rendimiento académico.

Tabla 7

Frecuencias y porcentajes para la variable rendimiento según cada nivel definido para la variable autoconcepto general

\begin{tabular}{lcccccc}
\hline \multicolumn{1}{c}{ Niveles } & \multicolumn{2}{c}{ Bajo } & \multicolumn{2}{c}{ Medio } & \multicolumn{2}{c}{ Alto } \\
\hline \multirow{3}{*}{ Bajo } & $\mathbf{f}$ & $\boldsymbol{\%}$ & $\mathbf{f}$ & $\boldsymbol{\%}$ & $\mathbf{f}$ & $\boldsymbol{\%}$ \\
\cline { 2 - 8 } Medio & 5 & 4,24 & 4 & 3,39 & 4 & 3,39 \\
Alto & 13 & 11,02 & 14 & 11,86 & 11 & 9,32 \\
& 10 & 8,47 & 32 & 27,12 & 25 & 21,19 \\
\hline
\end{tabular}

Por otro lado, en la relación entre rendimiento académico y autoconcepto académico, Tabla 8, se observan resultados especial llamativos, con 11 alumnos de rendimiento bajo ubicados en el nivel de autoconcepto académico bajo, por lo que las frecuencias y porcentajes reflejan una relación estrecha entre rendimiento académico bajo y nivel de autoconcepto académico bajo. Por el contrario, los resultados para el resto de niveles guardan cierta similitud. La media de los alumnos y alumnas de rendimiento bajo ha sido de 1,79 , desviación típica de, 74 , para el rendimiento medio de 2,60, desviación típica de ,61, y para el rendimiento alto de 2,48, desviación típica de ,64. Así, en la prueba Chi-cuadrado, valor de 25,47 significatividad de ,00, se han observado diferencias estadísticamente significativas. El índice de correlación de Pearson entre ambas variables ha sido de -,32, con una significatividad de ,00. Se demuestra que existe significatividad entre variables, es decir, hay relación entre rendimiento académico y autoconcepto académico, tal y como se ha señalado estas diferencias se dan en la asociación entre ambas variables para los niveles más bajos.

Tabla 8

Frecuencias y porcentajes para la variable rendimiento según cada nivel definido para la variable autoconcepto académico

\begin{tabular}{lcccccc}
\hline \multicolumn{1}{c}{ Niveles } & \multicolumn{2}{c}{ Bajo } & \multicolumn{2}{c}{ Medio } & \multicolumn{2}{c}{ Alto } \\
\hline \multirow{3}{*}{ Bajo } & $\mathbf{f}$ & $\boldsymbol{\%}$ & $\mathbf{f}$ & $\boldsymbol{\%}$ & $\mathbf{f}$ & $\boldsymbol{\%}$ \\
\cline { 2 - 8 } Medio & 11 & 9,32 & 3 & 2,54 & 3 & 2,54 \\
Alto & 12 & 10,17 & 14 & 11,86 & 15 & 12,71 \\
\hline
\end{tabular}

En las relaciones entre la edad de los escolares y el autoconcepto general (prueba Chi-cuadrado, valor de 106,53 significatividad de ,44, e índice de correlación de 
Pearson, valor de -.02, significatividad de ,81) y entre edad y autoconcepto académico (prueba Chi-Cuadrado, valor de 39,96 significatividad de ,56, e índice de correlación de Pearson, valor de -,04 significatividad de ,69), no se observan diferencias estadísticamente significativas.

Por último, se especifican los resultados en función del curso de los alumnos y alumnas participantes para las variables autoconcepto general (prueba Chi-Cuadrado, valor de 66,84 significatividad de ,58, e índice de correlación de Pearson, valor de -, 13 significatividad de ,78) y autoconcepto académico (prueba Chi-Cuadrado, valor de 31,00 significatividad de ,32, e índice de correlación de Pearson, valor de -,24 significatividad de ,81). Se obtiene una carencia de diferencias estadísticamente significativas.

\section{Discusión y conclusiones}

Tras el análisis de resultados se ha comprobado que los sujetos participantes presentan en general unos niveles altos autoconcepto general y autoconcepto académico. Se ha corroborado también que existe una correlación positiva entre autoconcepto, general y académico, y rendimiento académico, siendo el alumnado de menor rendimiento el que puntúa más bajo en autoconcepto, sobre todo en su dimensión académica. De este modo, se confirman tanto la hipótesis de partida como los resultados de las investigaciones previas ya citadas en el sentido de que el autoconcepto es una variable influyente en el rendimiento académico. Así, este constructo podría ser una de las causas del pobre desempeño del alumnado español en los informes PISA y también podría estar dificultando la detección y diagnóstico de las altas capacidades intelectuales.

Respecto a la cuestión de los más capaces, existe relación entre bajo autoconcepto general y existencia de altas capacidades intelectuales. Sin embargo, no existe significatividad entre autoconcepto académico y alta capacidad detectada. Por otro lado, si relacionamos ambos tipos de autoconcepto con la alta capacidad detectada y con el sexo de forma simultánea, tampoco aparece significatividad.

En cuanto a la cuestión de género, se ha visto que hay más niños que niñas en los niveles más altos de autoconcepto general y autoconcepto académico, si bien el análisis estadístico de datos ha concluido que estos resultados no son estadísticamente significativos. A pesar de ello, es importante no perder de vista la perspectiva de género ya que es una variable influyente en el autoconcepto según distintos autores (Amezcua, y Pichardo, 2000; Padilla, García, y Suárez, 2010; Pastor, Balaguer, y García-Merita, 2003). En el resto de variables de la investigación, edad y curso de los participantes, no han aparecido diferencias estadísticamente significativas.

Con todo ello, se hace evidente la necesidad de trabajar desde las aulas el autoconcepto del alumnado, pues está comprobado que una intervención específica mejora este constructo (Cerrillo, 2002), lo que, como ha quedado demostrado, terminará influyendo positivamente en otras variables, caso del rendimiento académico. Y para que las propuestas de intervención en estos ámbitos no queden únicamente en teorías, iniciativas puntuales o actividades a libre voluntad por parte del propio docente, sería interesante incluir en los marcos legales en materia educativa, no solo propuestas curriculares para desarrollar los procesos cognitivos del alumnado, sino también los procesos afectivos y emocionales. Está demostrado que el plano cognitivo y el plano emocional no deben separarse, pues son procesos complejos que suceden 
interconectados (Blanco, De Caso, y Navas, 2013). Además, debido a esta complejidad, se hace necesaria la demanda de nuevas investigaciones en torno al tema que permitan fundamentar y sentar sus bases educativas. Así, la educación emocional, que en ocasiones es relegada a un segundo plano, debería jugar un papel más importante en los currículos y contenidos académicos. De este modo estaremos avanzando un paso más hacia la consecución de una educación integral.

\section{Referencias}

Al-Srour, N.H., \& Al-Oweidi, A.M. (2016). Self-concept among Gifted and Non-gifted Students and its Relationship with Gender Variable in a Jordanian Sample. International Journal of Educational Sciences, 12(1), 50-56. doi: 10.1080/09751122.2016.11890412

Amezcua, J.A., \& Pichardo, M.C. (2000). Diferencias de género en autoconcepto en sujetos adolescentes. Anales de Psicología, 16(2), 207-214. Retrieved from https://www.um.es/analesps/v16/v16_2/10-16_2.pdf

Ancillo, I., Artola, T., Barraca, J., Pina, J., \& Mosteior, P. (2001). Diferencias de género y autoconcepto en alumnos de altas capacidades. En La educación de mujeres y varones en el tercer milenio: entre el código genético y social. In III Symposium Internacional sobre la Educación Femenina y Masculina en el Tercer Milenio, Centro Universitario Villanueva, Universidad Complutense de Madrid. Retrieved from https://www.researchgate.net/publication/275273138_Diferencias_de_genero_y _autoconcepto_en_Alumnos_de_Altas_Capacidades

Blanco, J., De Caso, A.M., \& Navas, G. (2013). Variables personales implicadas en el aprendizaje: ¿motivación y/o emoción? International Journal of Developmental and Educational Psychology, 1(1), 505-512. Retrieved from http://infad.eu/RevistaINFAD/2013/n1/volumen1/INFAD_010125_505-512.pdf

Cerrillo, M.R. (2002). Mejorar el autoconcepto en alumnos de un entorno desfavorecido. Revista de Psicodidáctica, 14, 71-85. Retrieved from http://www.ehu.eus/ojs/index.php/psicodidactica/article/view/145/141

Díaz-Aguado, M.J., \& Martínez-Arias, R. (1995). La evaluación de la adaptación socioemocional a través del autoinforme. El autoconcepto y la empatía. In M.J. Díaz-Aguado, $y \quad R$. Martínez-Arias (Ed.), Niños con dificultades socioemocionales. Instrumentos de evaluación (pp. 3-48). Madrid: Ministerio de Asuntos Sociales, Sistema de Atención Social a la Infancia (SASI).

Epstein, J.H. (1985). Review of Pier-Harris Children's Self-concept Scale (The way I feel about myself). In J.V. Mitchell (Ed.), The Ninth Mental Measurements Yearbook (pp. 1167-1169). Lincoln, United States: The University of Nebraska Press.

García, R. (2014). Diseño y validación de un instrumento de evaluación de la competencia matemática. Rendimiento matemático de los alumnos más capaces. Tesis de Doctorado para la obtención del título de Doctor en Educación. Facultad de Educación. UNED. Retrieved from http://espacio.uned.es/fez/view/tesisuned:Educacion-Rgarcia

García, R., Almeida, L.S., \& Viseu, F. (2017). El rendimiento matemático excelente en las evaluaciones PISA: Resultados para España y Portugal. Revista de Estudios e Investigación en Psicología y Educación, Extr. (1), 303-308. doi: 10.17979/reipe.2017.0.01.2998 
Guillén, F., \& Ramírez, M. (2011). Relación entre el autoconcepto y la condición física en alumnos del Tercer Ciclo de Primaria. Revista de Psicología del Deporte, 20(1), 45-59.

Retrieved from

http://www.psicologiadeporte.ulpgc.es/docs/Relacion-entre-autoconcepto-ycondicion-fisica-en-alumnos-del-tercer-ciclo-de-primaria-de-un-centroescolar.pdf

Jiménez, C. (2014). El desarrollo del talento: educación y alta capacidad. Madrid: Universidad Nacional de Educación a Distancia. Retrieved from https://canal.uned.es/mmobj/index/id/21559 .

Jiménez, C., \& García, R. (2013). Los alumnos más capaces en España. Normativa e incidencia en el diagnóstico y la educación. Revista Española de Orientación y Psicopedagogía, 24(1) 7-24. Retrieved from https://www2.uned.es/reop/pdfs/2013/24-1\%20-\%20Jimenez.pdf

Kerr, B. (1999). Orientación profesional de las mujeres superdotadas. En J. Ellis, y J. Willinsky, (1999). Niñas, Mujeres y Superdotación. Un desafio a la discriminación educativa de las mujeres (pp. 155-163). Madrid: Narcea.

Ministerio de Educación, Cultura y Deporte (2018). Estadísticas no universitarias. Retrieved from http://www.mecd.gob.es/servicios-al-ciudadanomecd/estadisticas/educacion/no-universitaria.html

Naranjo, M.L. (2007). Autoestima: un factor relevante en la vida de la persona y tema esencial del proceso educativo. Revista electrónica actualidades investigativas en educación, 7(3), 1-27. Retrieved from https://www.redalyc.org/pdf/447/Resumenes/Resumen_44770311_1.pdf

Navarro, E., Tomás, J.M., \& Oliver, A. (2006). Factores personales, familiares y académicos en niños y adolescentes con baja autoestima. Boletín de Psicología, 88, 7-25. Retrieved from https://www.uv.es/seoane/boletin/previos/N88-1.pdf

Organización para la Cooperación y el Desarrollo Económicos (2005a). PISA 2000. Resultados en España del estudio PISA 2000. Conocimientos y destrezas de los alumnos de 15 años. Retrieved from https://www.mecd.gob.es/inee/dam/jcr:eb6fde04-a7c1-465a-88b4e51465383807/pisa2000infnacional.pdf.

Organización para la Cooperación y el Desarrollo Económicos (2005b). PISA 2003. Matemáticas. Informe español. Retrieved from https://www.mecd.gob.es/inee/dam/jcr:60f4210c-d96d-43a2-8bc905fd881e82f7/informe-espanol-pisa-era-2009-3.pdf.

Organización para la Cooperación y el Desarrollo Económicos (2007). PISA 2006. Programa para la evaluación internacional de los alumnos. Informe Español. $\begin{array}{lllll}\text { Resultados } y & \text { contexto. } & \text { Retrieved }\end{array}$ https://www.mecd.gob.es/inee/dam/jcr:699d43f6-ddcc-4c7e-b7bfc0e0c288e949/pisainforme2006.pdf.

Organización para la Cooperación y el Desarrollo Económicos (2010). PISA 2009. Programa para la evaluación internacional de los alumnos. Informe Español. Resultados $y$ contexto. Retrieved from https://www.mecd.gob.es/inee/dam/jcr:bc05a3ce-effe-425b-a79bc92f0d43f8d1/pisa-2009-con-escudo.pdf.

Organización para la Cooperación y el Desarrollo Económicos (2014). PISA 2012. Programa para la evaluación internacional de los alumnos. Informe Español. $\begin{array}{llll}\text { Resultados } & y & \text { contexto. } & \text { Retrieved from }\end{array}$ https://www.mecd.gob.es/inee/evaluaciones-internacionales/pisa/pisa-2012.html. 
Organización para la Cooperación y el Desarrollo Económicos (2015). PISA in Focus ¿Qué subyace bajo la desigualdad de género en educación? Retrieved from http://www.mecd.gob.es/dctm/inee/pisainfocus/pif49espfinal.pdf?documentId=0901e72b81c7fb18.

Organización para la Cooperación y el Desarrollo Económicos (2016). PISA 2015. Programa para la evaluación internacional de los alumnos. Informe Español. Retrieved from https://www.mecd.gob.es/inee/evaluacionesinternacionales/pisa/pisa-2015.html.

Padilla, M.T., García, M.S., \& Suárez, M. (2010). Diferencias de género en el autoconcepto general y académico de estudiantes de $4^{\circ}$ de ESO. Revista de Educación, 352, 495-515. Retrieved from http://www.revistaeducacion.educacion.es/re352/re352_22.pdf

Pastor, Y., Balaguer, I., \& García-Merita, M.L. (2003). El autoconcepto y la autoestima en la adolescencia media: análisis diferencial por curso y género. Revista de Psicología social, 18(2), 141-159. Retrieved from https://dialnet.unirioja.es/servlet/articulo?codigo $=440864$

Pfeiffer, S. I. (2015). El Modelo Tripartito sobre la alta capacidad y las mejores prácticas en la evaluación de los más capaces. Revista de Educación, 368, 6695. http://redined.mecd.gob.es/xmlui/bitstream/handle/11162/111093/2015_\%20Rev istaEducacion368_66_Pfeiffer.pdf?sequence $=1 \&$ is Allowed $=y$

Puigcerver, M.J. (2003). Evaluación del Autoconcepto, Actitudes y Competencia Social en Sujetos Sordos. Tesis de doctorado para la obtención del título de Doctora en Psicología, Universidad de Murcia. Retrieved from http://www.culturasorda.org/wp-content/uploads/2015/04/Tesis-PuigcerverHurtado-2003.pdf

Rodríguez, S. (1982). Factores de rendimiento escolar. Barcelona: Oikos-Tau.

Stoeger, H., Balestrini, D.P., \& Ziegler, A. (2018). International perspectives and trends in research on giftedness and talent development. In S. I. Pfeiffer, E. ShaunessyDedrick, \& M. Foley-Nicpon (Eds.), APA handbook of giftedness and talent (pp. 25-37). Washington: American Psychological Association.

Trillo, M.C. (2012). Alta capacidad y género. Factores diferenciadores cognitivos y de personalidad en niños y niñas de alta capacidad. (Tesis doctoral). Universidad de Córdoba.

Retrieved

from https:/helvia.uco.es/xmlui/bitstream/handle/10396/7975/2012000000566.pdf?se quence $=3 \&$ is Allowed $=y$

Zeidner, M., \& Shani-Zinovich, I. (2015). A comparison of multiple facets of selfconcept in gifted vs. non-identified Israeli students. High Ability Studies, 26(2), 211-226. Retrieved from https://www.tandfonline.com/doi/full/10.1080/13598139.2015.1095076

Fecha de recepción: 07/03/2019

Fecha de revisión: 03/06/2019

Fecha de aceptación: 19/6/2019 